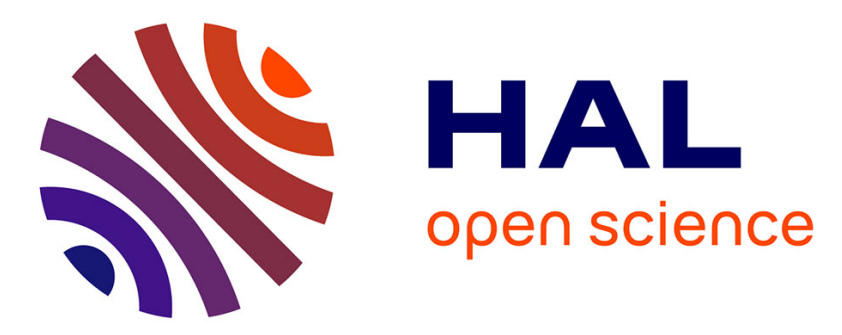

\title{
Reversible and dynamical control of aggregation and soft adhesion of T-responsive polymer-coated colloids
}

Giuseppe Boniello, Jérémy Malinge, Christophe Tribet, Emmanuelle Marie, Dražen Zanchi

\section{- To cite this version:}

Giuseppe Boniello, Jérémy Malinge, Christophe Tribet, Emmanuelle Marie, Dražen Zanchi. Reversible and dynamical control of aggregation and soft adhesion of T-responsive polymer-coated colloids. Colloids and Surfaces A: Physicochemical and Engineering Aspects, 2017, 532, pp.510-515. 10.1016/j.colsurfa.2017.04.011 . hal-01510328

\section{HAL Id: hal-01510328 \\ https: / hal.sorbonne-universite.fr/hal-01510328}

Submitted on 19 Apr 2017

HAL is a multi-disciplinary open access archive for the deposit and dissemination of scientific research documents, whether they are published or not. The documents may come from teaching and research institutions in France or abroad, or from public or private research centers.
L'archive ouverte pluridisciplinaire HAL, est destinée au dépôt et à la diffusion de documents scientifiques de niveau recherche, publiés ou non, émanant des établissements d'enseignement et de recherche français ou étrangers, des laboratoires publics ou privés. 


\title{
Reversible and dynamical control of aggregation and soft adhesion of T-responsive polymer-coated colloids
}

\author{
Giuseppe Boniello ${ }^{a}$, Jérémy Malinge ${ }^{\mathrm{a}}$, Christophe Tribet $^{\mathrm{a}}$, Emmanuelle \\ Marie $^{\mathrm{a}}$, Dražen Zanchi ${ }^{\mathrm{a}, \mathrm{b}, *}$ \\ ${ }^{a}$ Ecole Normale Supérieure-PSL Research University, Dpt Chimie, Sorbonne Universités - \\ UPMC Univ. Paris 06, CNRS UMR 8640, 24 rue Lhomond, 75005 Paris, France \\ ${ }^{b}$ Université de Paris VII Denis Diderot, 5 Rue Thomas Mann, 75013 Paris, France
}

\begin{abstract}
Colloids with aggregation and adhesion properties reversibly tunable by shift of $\mathrm{pH}, \mathrm{T}$, light etc. can be designed by deposition of stimuli-responsive polymer chains on the particle surface. The aim of this work was to investigate how to control the strength of temperature-triggered attraction by analysing self-aggregation kinetics and soft adhesion of colloids to a flat substrate. In order to endow the colloids with reversible and temperature-controlled interactions, silica or polystyrene microbeads $(d=200 \mathrm{~nm}$ and $1 \mu \mathrm{m})$ were coated by mixed solutions of poly(lysine)-grafted-polyethylenoxide (PLL-g-PEG, for steric repulsion) and PLL-g-PNIPAM (i.e. PLL with poly-N-isopropylacrylamide Tresponsive side chains). PEG-coated particles were stable in suspension, while the presence of PNIPAM provoked, at $T>T_{c}=32 \pm 1^{\circ} \mathrm{C}$, reversible aggregation and/or adsorption on glass plates. Dynamic light scattering following a T-jump from $25^{\circ} \mathrm{C}$ to $40^{\circ} \mathrm{C}$ was used to measure the aggregation rate and corresponding stability ratio $W$. For a molar fraction of PLL-g-PNIPAM, $f$, ranging from $100 \%$ down to about $20 \%$, particles aggregate rapidly with slowly increasing $W$. Below $f \approx 20 \%, W$ increases by 3 orders of magnitude. The real-time $2 \mathrm{D}$ tracking method was used to monitor $(x, y)$ positions of particles in suspension above microscope glass slides during a T-triggered adsorption. In
\end{abstract}

\footnotetext{
* Corresponding author

Email address: zanchi@ens.fr (Dražen Zanchi)
} 
order to capture transitory dynamics near PNIPAM collapse transition, particles tracks were recorded within a T-ramp of $10^{\circ} \mathrm{C} / \mathrm{min}$ from below to above $T_{c}$. The particle-substrate interaction was found to hinder the near-wall diffusion and provoke the soft adhesion, as revealed by observation of characteristic confined Brownian motion. Resulting confinement potential stiffness profile $\alpha(f)$ presents a crossover from constant to linearly increasing at $f \approx 20 \%$. Altogether, the characteristic coverage $f^{*} \approx 20 \%$ is interpreted as a crossover from discrete to continuous coverage pattern within the soft contact domain.

Keywords: Colloids, temperature responsive polymers, PNIPAM, soft adhesion, Brownian motion, aggregation

\section{Introduction}

Self-association and soft adsorption dynamics of polymer-coated colloids attract considerable attention in studies of bottom-up designed composite materials [1], colloidal crystals [2], bacterial adhesion [3], biofouling resistant de-

5 vices [4] etc... Controlled interactions with the solid phase can be also involved in a field-flow separation approach [5, 6. Reversible and dynamically tunable, or even time-modulated, interactions are prerequisite for other, far-fromequilibrium colloidal assemblies [7, 8]. External stimuli typically used for tuning surface, dynamical and/or interactions in colloids are light [9], electric [10] and magnetic [11] fields, $\mathrm{pH}$ [12, 13, 14], solvent [15, 16] or temperature variation [17]. Tunable aggregation and/or adhesion, triggered by external stimuli, may entail benefits for the uprising class of functional materials. In this respect, stimuli-responsive colloidal particles find applications in controlled stabilization, destabilization and inversion of emulsions, foams and suspensions [18, in tun15 able catalysis [19, 20], and in temperature-sensitive colloidosomes [21].

In the present paper we report results on self-aggregation and soft adsorption on flat surface of colloids endowed with T-responsive polymer coating with controlled interaction strength. The temperature of the sample is easily controlled and tuned by using thermostated cells (for particle-particle study) or 
Peltier elements and heating stages. On the other side stimuli-responsive polymer coating can be used as an efficient mean to control their surface properties and induce stimuli-sensitive behaviour [22]. Since we are interested in temperature-dependent mechanisms Poly(N-isopropylacrylamide) (PNIPAM) is used. PNIPAM-coated particles undergo temperature-triggered collapse transition above a critical temperature $T_{c} \sim 32^{\circ} \mathrm{C}[22,23,24,25]$. A robust experimental strategy enabling a facile preparation of coated particles has been proposed in a recent work [26]. The method consists in the use of cationic comb-like poly(Lysine) derivative as a versatile platform to deposit functional strands on anionic particles. The PLL-graft-PEO has been extensively studied (and is now commercialized) for its excellent bio-repellency that is effective on a broad variety of surfaces (metals covered by a thin oxide layer, plasma-cleaned polymers including PS and PDMS, glass, ceramic, etc). We here broaden the family of this interesting polymer, by addition of T-responsiveness. It is likely that other polymers (diblock) may achieve similar properties, but it is not always the case: for instance, alternative architectures (such as grafted poly(ethyleneimine)) make poor adlayers, prone to locally attractive (cationic) micro-defects. The polycationic PLL backbone ensures tight Coulomb attachment onto anionic surfaces (glass, plastics, silicium) [4, 27, 28, 29]. Different side chains can be grafted on such PLL backbones. For the purposes of the present work we used poly-N40 isopropylacrylamide (PNIPAM) and polyethylenoxide (PEG). PLL-g-PNIPAM, provides the thermo-responsive behaviour required for reversibly switched colloidal interactions. PLL-g-PEG endows surfaces with steric repulsive shield in the whole range of working temperatures. At $T>T_{c} \sim 32^{\circ} \mathrm{C}$ PNIPAM chains collapse to give way to particle-particle and particle-substrate attraction. The

45 strength of this interaction is itself tunable by adjusting PLL-g-PNIPAM molar ratio $f$ in the coating. Thus, our system is determined by two control parameters $T$ and $f$.

The aim of the present analysis is to explore the possibilities to control the strength of the T-triggered interactions between polymer-coated colloids. We used dynamic light scattering to monitor the aggregation kinetics following a 
T-jump from $25^{\circ} \mathrm{C}$ to $40^{\circ} \mathrm{C}$. Aggregation rate in the very beginning of kinetics allowed us to extract the stability ratio $W$. The adsorption of particles on a flat surface in a slow T-ramp was studied by $2 \mathrm{D}$ particle-tracking using a microscope and a camera. The resulting tracks were analysed to yield the mean 55 square displacement (MSD), which, in turn, was fitted to the Langevin equation of a Brownian particle in harmonic confining potential. In Section 2 we describe materials and methods. Section 3 reports on the particle-particle interactions, i.e. the colloid destabilisation - re-dispersion cycles in a series of ascending descending $T$-ramps and the DLS results in T-jump experiments. Section 4 focuses on the particle-surface interactions leading to the adsorption process and subsequent Brownian dynamics of softly adhered beads. Section 5 contains discussion of the $f$ dependence of aggregation stability ratio $W$ and soft adhesion stiffness $\alpha$. Conclusions and implications of our results are given in Section 6 .

\section{Materials and methods}

Particles self-association. Commercial carboxylated polystyrene microspheres (Polybead, Polyscience, $200 \mathrm{~nm}$ in diameter) were dialysed against water (SlideA-Lyzer, $M_{W}$ cutoff $3500 \mathrm{kDa}$, Thermo Scientific). The solution was diluted in $5 \mathrm{mM}$ phosphate buffer. Particle coating was obtained mixing $13 \mu \mathrm{L}$ of beads in PBS with $60 \mu L$ of polymer solution $10 g . L^{-1}$ ( $f \%$ PLL-g-PNIPAM, $(100-f) \%$ PLL-g-PEG) in $5 \mathrm{mM}$ phosphate buffer. (PLL: $M_{w}=15000-30000 \mathrm{~g} \cdot \mathrm{mol}^{-1}$, grafting density 20\%; PEG: $M_{w}=2000 \mathrm{~g} \cdot \mathrm{mol}^{-1}, R_{g}=1.8 \mathrm{~nm}$; PNIPAM: $\left.M_{w}=2000 \mathrm{~g} \cdot \mathrm{mol}^{-1}, R_{g}=1.8 \mathrm{~nm}\right)$. Polymer chains adsorb on the particle surface up to saturation density (typically of the order of $1.1 \mathrm{mg} \mathrm{m}^{-2}$ [29, 4], not measured for present system). The resulting suspension was incubated for 5 min. Polymer excess was removed by size exclusion chromatography (Sephacryl HR 300 column, $2.5 \times 1.5 \mathrm{~cm}$ ) pre-equilibrated with $5 \mathrm{mM}$ phosphate buffer. Typical retention volume of polymer-coated beads was $1.1 \mathrm{~mL}$, making the beads well separated from excess unbound polymer (retention volume of $2.4 \mathrm{~mL}$ ). The efficiency of the protocol to obtain a controlled $f$ has been ensured by fluores- 
80 the PEG-to-PNIPAM ratio in coating remains indeed the same as the one in the co-polymer solution before adsorption. The $1 \mathrm{~mL}$ samples prepared at room temperature $\left(T=25^{\circ} \mathrm{C}\right)$ were plunged in the thermostated cell $\left(T=40^{\circ} \mathrm{C}\right)$ of DLS apparatus (Brookhaven BI-200SM equipped with a multiple tau digital correlator and a $30 \mathrm{~mW}$ diode laser operating at $637 \mathrm{~nm}$ ). The scattered light intensity was measured at $90^{\circ}$ scattering angle. Temperature control within $0.1^{\circ} \mathrm{C}$ was achieved using a bath circulator connected to a temperature probe plunged in the decalin bath nearby the sample cell. The temperature of the sample attains $32^{\circ} \mathrm{C}$ within seconds and the aggregation takes place. The effective hydrodynamic diameter (second cumulant) was monitored in time. $\zeta$-potential were measured in a Zetasizer (Nano ZS, Malvern Instruments Ltd) equipped with a He-Ne laser and temperature controller $\left( \pm 0.1^{\circ} \mathrm{C}\right)$. Five measurements were recorded and their standard deviation is reported.

Particles adsorption on a flat substrate. Silica beads $(0.96 \mu \mathrm{m}$ in diameter, replacing the supernatant with deionised water.

The flat substrate consisted in a borosilicate glass microscope slide (thickness $=0.17 \mathrm{~mm}$ ) cleaned with ethanol and sonicated for $30 \mathrm{~min}$ in a $1 \mathrm{M}$ sodium hydroxide. After rinsing with deionised water, the experimental cell was prepared by superposing bi-adhesive tape and a mylar film. A $52 \mathrm{~mm}$ x $5 \mathrm{~mm}$ $110 \times 50 \mu \mathrm{m}$ channel was thus created between the borosilicate glass slide and the 
mylar film. The bottom of the channel was coated with PLL-g-PEG $\left(M_{w}=\right.$ $\left.20000 \mathrm{~g} \cdot \mathrm{mol}^{-1}\right)$ by injecting co-polymer solution $\left(1 \mathrm{~g} \cdot \mathrm{L}^{-1}\right.$ in $10 \mathrm{mM}$ phosphate buffer) in the cell and left to incubate for $30 \mathrm{~min}$. The cell was then rinsed with deionised water and dried by compressed air. Note that the flat substrate thereby prepared was not thermo-sensitive and had no active role in particle adhesion. In fact, the full PLL-g-PEG coating ensured a steric repulsion with particles of whatever the coating when the T-stimulus is absent. The T-ramp experiments were performed using a temperature controlled microscope stage (Linkam PE94). The particles suspension were injected into the cell at $T=26^{\circ} \mathrm{C}$ and subjected to a temperature ramp of $10^{\circ} \mathrm{C} / \mathrm{min}$ up $T=36^{\circ} \mathrm{C}$. The beads are observed by Phase Contrast Microscopy (Leica DM IRE 2) equipped with objective (Leica HCX Fluotar L 63x/0.70) and a CMOS camera (iDS uEye CP, pixel size $=0.12 \mu \mathrm{m})$. Particles were tracked in real time by Pico-eye software [30] allowing sub-pixel resolution $(\sim 5 \mathrm{~nm})$ at 50 frames/s and the mean-square displacement (MSD) was extracted from particles track record using a home-made numerical routine. The MSD profiles were averaged over typically 5 different particles tracks.

\section{Particle-particle interaction}

Reversibility. Temperature-triggered aggregation, with emphasis on reversibility issue, was studied for $f=100 \%$ PLL-g-PNIPAM (complete particle surface is thermo-sensitive) by UV-vis spectroscopy, dynamic light scattering (DLS), and zetametry. An indicator of the aggregation is the abrupt decrease of transmittance at $650 \mathrm{~nm}$ at $T \simeq 32^{\circ} \mathrm{C}$ (blue line in Fig 1 1 ). DLS provides the same information from a quantitative point of view: the suspension is stable below $32^{\circ} \mathrm{C}$ (the initial diameter is kept constant at $200 \mathrm{~nm}$ ), larger aggregates showup above the transition temperature (red line in Fig 1a). Bare particles as well as these without PNIPAM $(f=0)[26$ ] were all stable in suspension at all experimentally accessible temperatures. Zetametry experiments, Fig,1p), confirmed the temperature-dependent surface properties. At $T<32^{\circ} \mathrm{C}$, the weakly 


$$
D_{h}(t)=D_{0}\left(1+\frac{\alpha t}{\tau W}\right)
$$

where $D_{h}(t)$ is the mean hydrodynamic diameter at time $t, D_{0}=200 \mathrm{~nm}$ 
is the initial particle diameter, $\alpha$ is a numerical pre-factor of the order of 1 , and $\tau=\frac{3 \eta}{4 k_{B} T c_{0}}$ is the universal diffusion limited aggregation time ( $\eta$ stands for

\section{Particle-substrate interaction and dynamics of softly confined par- ticles}

In this section we report the results obtained using a simple method to trigger in a controlled way the attractive interactions between the colloids and the flat substrate, which made possible the observation of Brownian dynamics before and after adsorption, including the very event of particle stopping. For this purpose we used real-time tracking within a temperature ramp. The advantage of temperature ramp experiments is that we are always sure to catch the conformational transition in PNIPAM, i.e. the repulsive-attractive switch on particle surface, even without a fine control on the cell temperature. Another advantage is that the moment of PNIPAM conformational transition can be estimated if many particles are in observation window: we are sure that this transition occurs just before the first particle is adsorbed. At the beginning of acquisition the temperature of the stage is $26^{\circ} \mathrm{C}$ and the T-ramp $10^{\circ} \mathrm{C} / \mathrm{min}$. allows for a comfortable acquisition, still being slow enough not to interfere with PNIPAM transition kinetics itself. In the beginning of T-ramp all particles undergo a free 
2D Brownian motion because of repulsive steric particle-surface interactions. At the end of acquisition the same particles are attached on the substrate, and free diffusion is not observed anymore. This behaviour is reversible, as observed in well $U(x)=\frac{1}{2} \alpha\left(x^{2}+y^{2}\right)$ :

$$
M S D(\tau)=\frac{k_{B} T}{\alpha}\left[1-\exp \left(-\frac{2 \alpha}{\gamma} \tau\right)\right],
$$

where $\gamma=6 \pi \eta R$ is the viscous friction, $\eta$ being the water viscosity. In Figure 5) the calculated best fitting curves are plotted together with $M S D(\tau)$ profiles extracted from the $(x, y)$ tracking. The corresponding values of the stiffness $\alpha(f)$

Once adsorbed on the surface, the particles have observable confined fluctuations, as seen in the inset of Fig 4. Fluctuations for particles adsorbed at the substrate at a constant temperature $T=36^{\circ} \mathrm{C}$ are recorded in real time by Picoeye software [30] (sub-pixel resolution $\sim 5 \mathrm{~nm}$, acquisition rate 50 frames $/ \mathrm{s}$ ) and the amount of PLL-g-PNIPAM on the particle surface. It is important to notice that the overall experimental noise (other than particle motion) in the $(x, y)$ track contributes by an amount certainly lower than (or equal to) the narrowest observed distribution $(f=100 \%)$. Consequently we are sure that any wider distribution are dominated by the stochastic dynamics of the bead softly attached to substrate. MSDs as a function of time lag $\tau$ for a range of PNIPAM coating fraction $f(\mathrm{Fig} / 5 \mathrm{~b})$ give a complete picture of particle fluctuations. At small timescales, MSDs are linear functions of $\tau$, signature of motion induced by thermal excitation. At longer times, MSDs at all particle coatings reach a free chain ends to the regions where PNIPAM went hydrophobic. The profile $M S D(\tau)$ and the confinement range (plateau position) strongly depend on $f$ : the strength of the anchoring is related to the number of PLL-g-PNIPAM blocks that are involved. For a more quantitative analysis, we fit the MSD proare shown in Fig 6 . 


\section{Discussion}

The temperature response of core-shell particles covered by PNIPAM, and reversibility (namely hysteresis) after excursion at high $\mathrm{T}$, has been regularly an issue in the control of solubility of particles. A recent report by Scherman [32] indicates that pure PNIPAM-AuNPs (i.e., no free PNIPAM in solution) do not aggregate, presumably because of intra-corona collapse at high surface coverage with PNIPAM. We point out that we purified our particles by similar means as Scherman, to remove free unbound polymers from the solutions. On the other hand, PNIPAM precipitation is typically reversible, but usually prone to large hysteresis (dissolution occurs at $\mathrm{T}$ lower by several ${ }^{\circ} \mathrm{C}$ than aggregation). This is not the case of our system, which suggest that inter-particle tight bridging is not as effective as with conventional long PNIPAM chains [33.

The presence of PNIPAM on the surface of a colloid is able to induce attraction at high temperature $\left(T \gtrsim T_{c}\right)$, which provokes the self-aggregation and substrate adhesion. In both cases, the molar ratio of PLL-g-PNIPAM which covers the surface (expressed by the parameter $f$ ) tunes the strength of the interaction. For particle aggregation this means that the effectiveness of sticky collisions, and consequently the kinetics of aggregation, is affected by $f$. In the case of particle adsorption $f$ changes the stiffness of the elastic trap where 245 the bead is confined. Here we will discuss the most important two results in this regard. The first result is that the self-association stability parameter as function of $f$ has two well distinguished regimes, delimited by the crossover at $f=f^{*} \simeq 20 \%$, see Fig. 3 . The second result is that the harmonic confinement potential stiffness $\alpha$ present a crossover at similar value of $f^{*}$, see Fig. 6. In both geometries two regimes are characterised as i) $f \lesssim f^{*} \simeq 20-25 \%$ where the properties slightly depend on $f$; ii) $f \gtrsim f^{*} \simeq 20-25 \%$, where the reference parameter ( $W$ for aggregation, $\alpha$ for adhesion) is linear in $f$. A tentative explanation comes from the possible arrangement on PLL-g-PNIPAM blocks on the bead. At low polymer concentration it is very likely that PLL-g-PNIPAM blocks would be isolated (Fig/7 ). A finite number of them would be involved in the 
aggregation/adsortion process. At high concentration a continuous distribution is obtained (Fig,7 7 ). The effect is proportional to the number of polymer blocks involved in the contact region. By using percolation argument an approximated threshold between the two regimes can be found around $f \simeq 25 \%$ (Fig.7 7 ), in agreement with experimental observations in both addressed systems.

Let's put our findings in the context of actual research on soft adhesion inspired by biological phenomena of the cell-surface adhesion or the receptordrug docking. The dynamics of surface adhesion of micro-particles in a shear flow has been recently studied by [34]. Interesting transient regime was found, between fast drift regime, in which particle is carried by the stream without any contact with the wall, and the arrest of the particle against the wall. In this intermediate regime the particle movement can be interpreted as rolling (or crawling) over the surface. The contact adhesion allowing for a rolling-and-noslipping constraint, is made possible by continuous attachment detachment of polymer-surface weak links. Interestingly, the theoretically studies of slipping and rolling of cells [35] and of soft adhesion by elasto-hydrodynamical effects [36] interpret the transient states as highly non-trivial phenomena in which the tether-ligand searching time, the rigidity of tethers, together with the lubrication effect in the narrow space between bead and the substrate determine collectively 275 the ageing dynamics in the very last moments before the soft attachment. The relevance of diffusion limited sticking events between polymer head and the substrate has been pointed out already in the context of binding between two surface-tethered reactants in relative motion 37 .

Cited works, focused mainly on the shear-driven adhesion, allow us to define some perspectives of the T-ramp approach. These works point out to the rolling (or crawling) phase of the adhesion in the shear flow. During this phase the particle-substrate contact domain explores a considerable fraction of the particle surface. If the flow is added during the sticky phase of our system, particles with stronger adhesion will roll less and vice-versa. The separation procedure could be further optimised by subjecting the system to a series of ascending and descending temperature jumps. 
Our experiment in T-ramp can be also compared to the experiments by Kumar et al.[38, in which the particle was initially held by an optical trap above a weakly sticky surface, released, and the subsequent motion was then

\section{Acknowledgments}

We thank Ken Sekimoto for stimulating and helpful discussions. This work was mainly supported by ANR DAPPlePur 13-BS08-0001-01. Authors benefited from access to zeta-potential measurement of "Institut Pierre-Gilles de 310 Gennes (program "Investissements d'Avenir" ANR-10-EQPX-34) with the help of J. Fattacioli. 


\section{References}

[1] J. Largo, P. Tartaglia, F. Sciortino, Effective nonadditive pair potential for lock-and-key interacting particles: The role of the limited valence, Physical Review E 76 (1) (2007) 011402. doi:10.1103/PhysRevE.76.011402.

[2] F. Sciortino, E. Zaccarelli, Reversible gels of patchy particles, Current Opinion in Solid State and Materials Science 15 (6) (2011) 246-253. doi:10.1016/j.cossms.2011.07.003.

[3] S. Gon, K.-N. Kumar, K. Nüsslein, M. M. Santore, How bacteria adhere to brushy peg surfaces: clinging to flaws and compressing the brush, Macromolecules 45 (20) (2012) 8373-8381. doi:10.1021/ma300981r.

[4] S. Gon, B. Fang, M. Santore, Interaction of cationic proteins and polypeptides with biocompatible cationically-anchored peg brushes, Macromolecules 44 (20) (2011) 8161-8168. doi:10.1021/ma201484h.

[5] J. C. Giddings, Field-flow fractionation: analysis of macromolecular, colloidal, and particulate materials, Science 260 (5113) (1993) 1456-1465. doi:10.1126/science.8502990.

[6] M. Martin, R. Beckett, Size selectivity in field-flow fractionation: Lift mode of retention with near-wall lift force, The Journal of Physical Chemistry A 116 (25) (2012) 6540-6551. doi:10.1021/jp212414e@proofing.

[7] R. Straube, M. Falcke, Reversible clustering under the influence of a periodically modulated binding rate, Physical Review E 76 (1) (2007) 010402. doi:10.1103/PhysRevE.76.010402.

[8] K. Bishop, B. Grzybowski, Localized chemical wave emission and mode switching in a patterned excitable medium, Physical review letters 97 (12) (2006) 128702. doi:10.1103/PhysRevLett.97.128702.

[9] C. Alvarez-Lorenzo, L. Bromberg, A. Concheiro, Light-sensitive intelligent drug delivery systems, Photochemistry and photobiology 85 (4) (2009) 848860. doi:10.1111/j.1751-1097.2008.00530.x 
[10] S. Murdan, Electro-responsive drug delivery from hydrogels, Journal of controlled release 92 (1) (2003) 1-17. doi:10.1016/S0168-3659(03)00303-1

[11] M. Zrinyi, Intelligent polymer gels controlled by magnetic fields, Colloid \& Polymer Science 278 (2) (2000) 98-103. doi:10.1007/s003960050017.

[12] V. Tsyalkovsky, R. Burtovyy, V. Klep, R. Lupitskyy, M. Motornov, S. Minko, I. Luzinov, Fluorescent nanoparticles stabilized by poly (ethylene glycol) containing shell for ph-triggered tunable aggregation in aqueous environment, Langmuir 26 (13) (2010) 10684-10692. doi:10.1021/ la101021t.

[13] M. Motornov, J. Zhou, M. Pita, I. Tokarev, V. Gopishetty, E. Katz, S. Minko, An integrated multifunctional nanosystem from command nanoparticles and enzymes, Small 5 (7) (2009) 817-820. doi:10.1002/ smll.200801550.

[14] M. Motornov, R. Sheparovych, R. Lupitskyy, E. MacWilliams, S. Minko, Responsive colloidal systems: Reversible aggregation and fabrication of superhydrophobic surfaces, Journal of colloid and interface science 310 (2) (2007) 481-488. doi:10.1016/j.jcis.2007.01.052

[15] M. Motornov, R. Sheparovych, R. Lupitskyy, E. MacWilliams, O. Hoy, I. Luzinov, S. Minko, Stimuli-responsive colloidal systems from mixed brush-coated nanoparticles, Advanced Functional Materials 17 (14) (2007) 2307-2314. doi:10.1002/adfm.200600934.

[16] F. Wang, L. Cheng, T. Chen, D. Zhu, Q. Wen, S. Wang, Facile preparation of polymeric dimers from amphiphilic patchy particles, Macromolecular rapid communications 33 (10) (2012) 933-937. doi:10.1002/marc. 201100787.

[17] J. M. Horton, C. Bao, Z. Bai, T. P. Lodge, B. Zhao, Temperature-and phtriggered reversible transfer of doubly responsive hairy particles between 
water and a hydrophobic ionic liquid, Langmuir 27 (21) (2011) 1332413334. doi:10.1021/la2031818.

[18] M. A. C. Stuart, W. T. Huck, J. Genzer, M. Müller, C. Ober, M. Stamm, G. B. Sukhorukov, I. Szleifer, V. V. Tsukruk, M. Urban, et al., Emerging applications of stimuli-responsive polymer materials, Nature materials 9 (2) (2010) 101-113. doi:10.1038/nmat2614.

[19] Y. Lu, Y. Mei, M. Drechsler, M. Ballauff, Thermosensitive core-shell particles as carriers for ag nanoparticles: modulating the catalytic activity by a phase transition in networks, Angewandte Chemie International Edition 45 (5) (2006) 813-816. doi:10.1002/anie.200502731.

[20] Y. Lu, S. Proch, M. Schrinner, M. Drechsler, R. Kempe, M. Ballauff, Thermosensitive core-shell microgel as a ìnanoreactorî for catalytic active metal nanoparticles, Journal of Materials Chemistry 19 (23) (2009) 3955-3961. doi:10.1039/B822673N

[21] D. B. Lawrence, T. Cai, Z. Hu, M. Marquez, A. Dinsmore, Temperatureresponsive semipermeable capsules composed of colloidal microgel spheres, Langmuir 23 (2) (2007) 395-398. doi:10.1021/la062676z.

[22] O. J. Cayre, N. Chagneux, S. Biggs, Stimulus responsive core-shell nanoparticles: synthesis and applications of polymer based aqueous systems, Soft Matter 7 (6) (2011) 2211-2234. doi:10.1039/C0SM01072C.

[23] M.-Q. Chen, T. Serizawa, M. Li, C. Wu, M. Akashi, et al., Thermosensitive behavior of poly (n-isopropylacrylamide) grafted polystyrene nanoparticles, Polymer journal 35 (12) (2003) 901-910. doi:10.1295/polymj.35.901.

[24] M.-Q. Zhu, L.-Q. Wang, G. J. Exarhos, A. D. Li, Thermosensitive gold nanoparticles, Journal of the American Chemical Society 126 (9) (2004) 2656-2657. doi:10.1021/ja038544z. 
[25] Q. Zhao, N. Chen, D. Zhao, X. Lu, Thermoresponsive magnetic nanoparticles for seawater desalination, ACS applied materials \& interfaces 5 (21)

[26] J. Malinge, F. Mousseau, D. Zanchi, G. Brun, C. Tribet, E. Marie, Tailored stimuli-responsive interaction between particles adjusted by straightforward adsorption of mixed layers of poly (lysine)-g-peg and poly (lysine)g-pnipam on anionic beads, Journal of colloid and interface science 461

[27] G. L. Kenausis, J. Vörös, D. L. Elbert, N. Huang, R. Hofer, L. RuizTaylor, M. Textor, J. A. Hubbell, N. D. Spencer, Poly (l-lysine)-g-poly (ethylene glycol) layers on metal oxide surfaces: attachment mechanism and effects of polymer architecture on resistance to protein adsorption,

$[29]$ adsorption using a patchy protein-resistant brush, Langmuir 26 (14) (2010) 12147-12154. doi:10.1021/la1016752.

415 [30] C. Gosse, V. Croquette, Magnetic tweezers: micromanipulation and force measurement at the molecular level, Biophysical journal 82 (6) (2002) 33143329. doi:10.1016/S0006-3495(02)75672-5.

[31] M. Von Smoluchowski, Drei vortrage uber diffusion. brownsche bewegung und koagulation von kolloidteilchen, Z. Phys. 17 (1916) 557-585. 

The importance of excess poly(n-isopropylacrylamide) for the aggregation of poly(n-isopropylacrylamide)- coated gold nanoparticles, ACS Nano 10 (2016) 3158-3165. doi:10.1021/acsnano.5b04083.

[33] B. Sun, Y. Lin, P. Wu, H. W. Siesler, et al., A ftir and 2d-ir spectroscopic study on the microdynamics phase separation mechanism of the poly (nisopropylacrylamide) aqueous solution, Macromolecules 41 (4) (2008) 15121520. doi:10.1021/ma702062h

[34] S. Kalasin, M. M. Santore, Near-surface motion and dynamic adhesion during silica microparticle capture on a polymer (solvated peg) brush via hydrogen bonding, Macromolecules 49 (1) (2015) 334-343. doi:10.1021/ acs.macromol.5b01977,

[35] C. Korn, U. Schwarz, Dynamic states of cells adhering in shear flow: from slipping to rolling, Physical review E 77 (4) (2008) 041904. doi:10.1103/ PhysRevE.77.041904

[36] M. Mani, A. Gopinath, L. Mahadevan, How things get stuck: Kinetics, elastohydrodynamics, and soft adhesion Phys. Rev. Lett. 108 (2012) 226104. doi:10.1103/PhysRevLett.108.226104. URL http://link.aps.org/doi/10.1103/PhysRevLett.108.226104

[37] K.-C. Chang, D. A. Hammer, The forward rate of binding of surfacetethered reactants: effect of relative motion between two surfaces, Biophysical Journal 76 (3) (1999) 1280-1292. doi:10.1016/S0006-3495(99) 77291-7

[38] D. Kumar, S. Bhattacharya, S. Ghosh, Weak adhesion at the mesoscale: 口 particles at an interface, Soft Matter 9 (2013) 6618-6633. doi:10.1039/ 445 C3SM00097D.

URL http://dx.doi.org/10.1039/C3SM00097D 


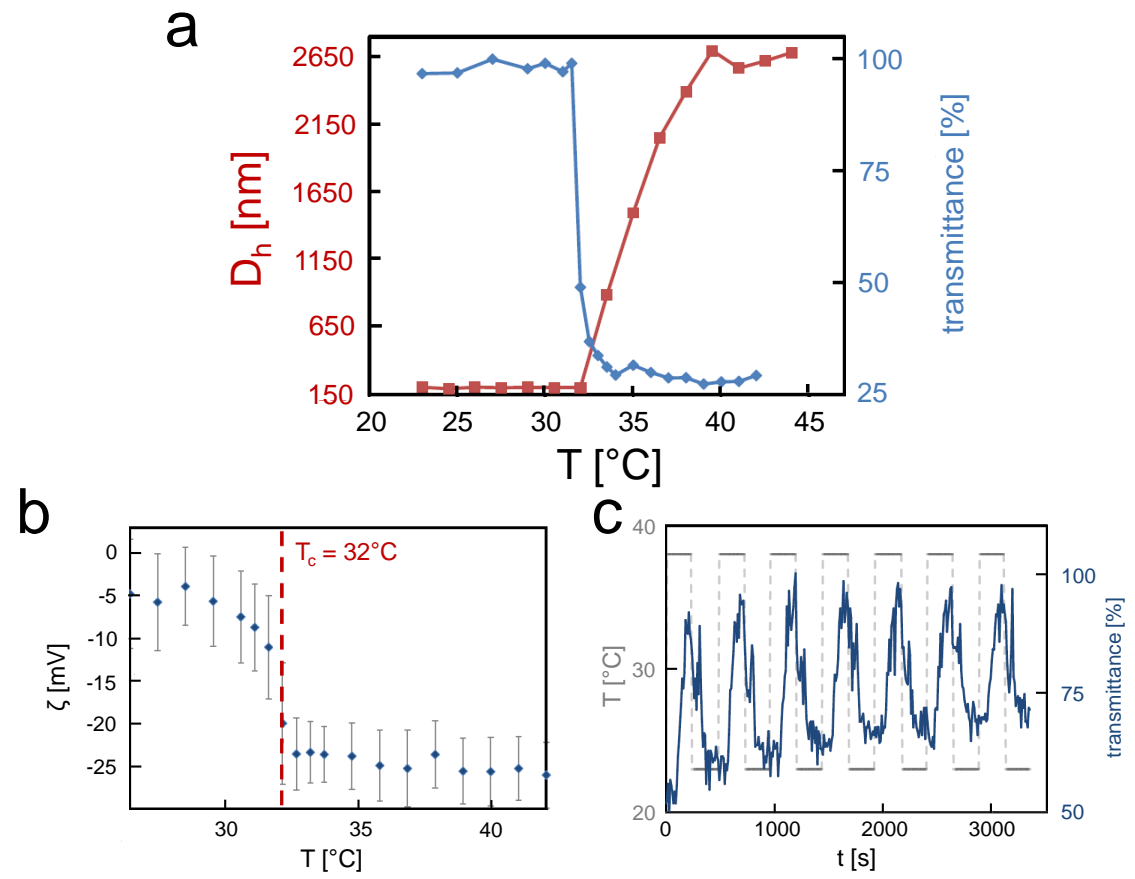

Figure 1: Temperature-triggered aggregation of coated $200 \mathrm{~nm}$ polystyrene beads. a. $100 \%$ PLL-g-PNIPAM coated particles show aggregation by the variation of the transmittance at $650 \mathrm{~nm}$ (blue line, right scale) and of the apparent hydrodynamic diameter measured by DLS at $90^{\circ}$ angle (red line, left scale). Both the measurements are recorded during $\mathrm{a}+1^{\circ} \mathrm{C}$ temperature increment every 4 minutes. b. $\zeta$-potential as a function of temperature for a bead saturated with PLL-g-PNIPAM (100\% PLL-g-PNIPAM). c. Transmittance at $650 \mathrm{~nm}$ for a dispersion of $100 \%$ PLL-g-PNIPAM coated beads during heating/cooling cycles. Figure adapted from [26]. 


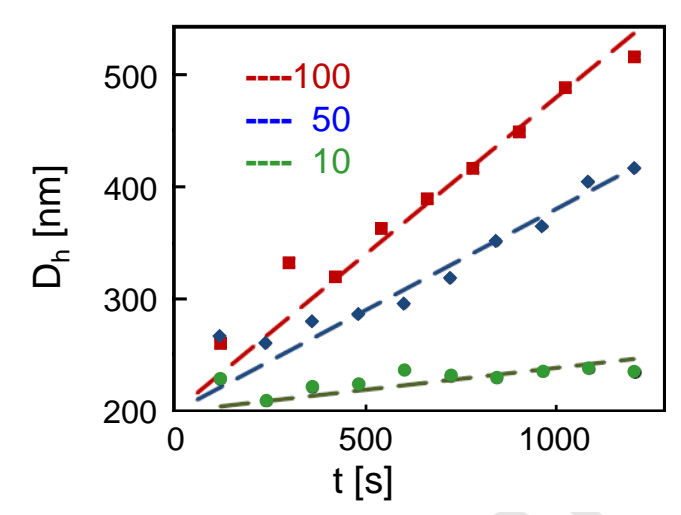

Figure 2: DLS measurements at $40^{\circ} \mathrm{C}$ for particles at PNIPAM fractions $f=100 \%$ (red squares), $50 \%$ (blue lozenges), $10 \%$ (green circles). Beads concentration $=8.5-10.5 \mu \mathrm{g} . \mathrm{L}^{-1}$ in $5 \mathrm{mM}$ phosphate buffer. Figure adapted from [26].

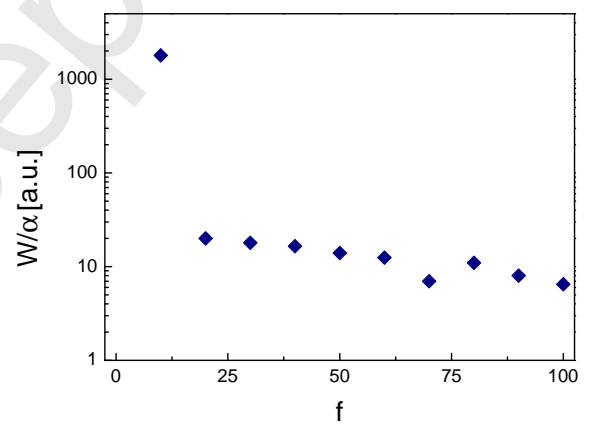

Figure 3: Stability ratio $W$, normalized by the numerical pre-factor $\alpha$, as a function of PLLg-PNIPAM surface fraction $f$. Figure adapted from [26]. 


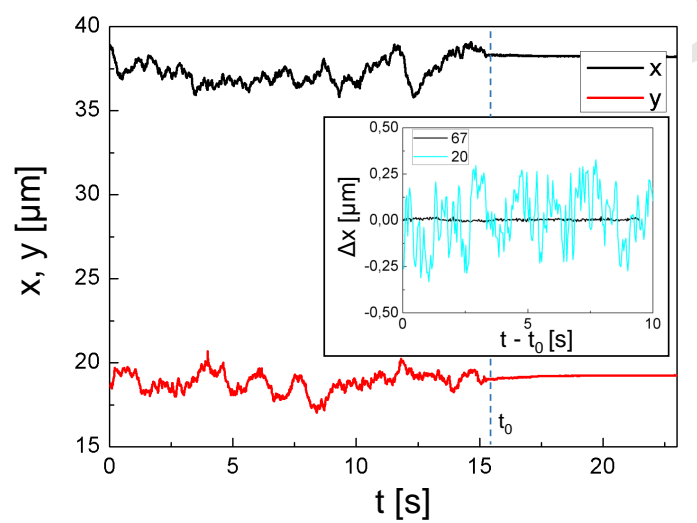

Figure 4: $\mathrm{x}-\mathrm{y}$ tracking of a silica bead coated with a mixture PLL-g-PEG/PLL-g-PNIPAM (PNIPAM fraction: $f=67 \%$ ) during an increasing temperature ramp $10^{\circ} \mathrm{C} / \mathrm{min}$ around the transition temperature. Transition between diffusive and adsorption regimes occurs at time $t_{0}$ (dashed line). Starting positions for $\mathrm{x}$ and $\mathrm{y}$ trajectories are arbitrary choices in the lab frame. Inset: detail of $\mathrm{x}$ profile in the adsorption regime. Two particle coatings $(f=67 \%, 20 \%)$ are shown for comparison. 
a

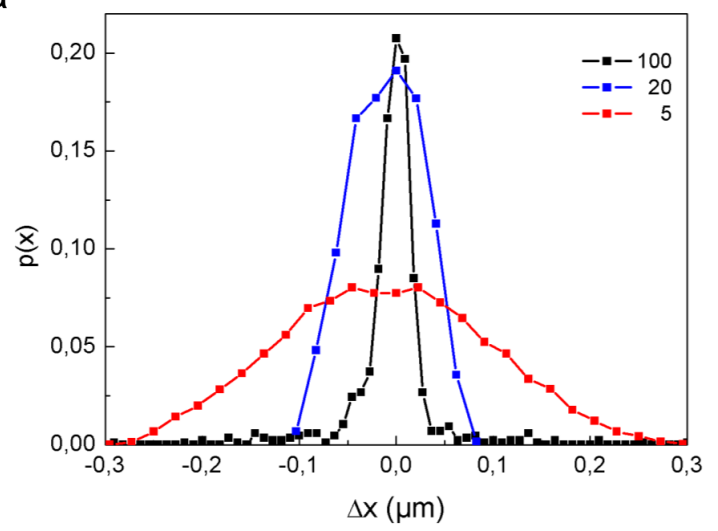

b

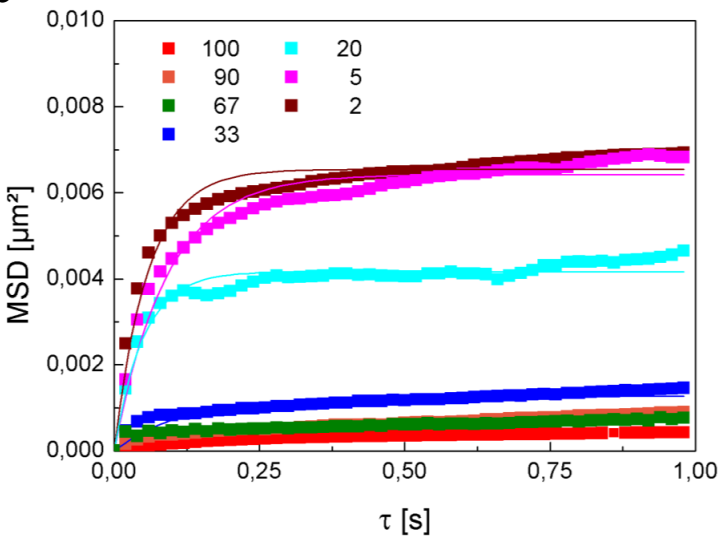

Figure 5: Confined fluctuations of softly attached particles a. Position distributions of particles adsorbed on the flat substrate: $f=100 \%$ (black), $f=20 \%$ (blue), $f=5 \%$ (red). Different widths suggest a f-dependent anchoring. b. MSD vs. time lag $\tau$ for adsorbed particles in the experimental range of $f$ values. Thin lines are fits to formula in Eq1 1 solution of Langevin equation in a harmonic potential well. The plateau value give us the "elasticity" constant $\alpha$, whose best fitting values are shown in Fig 6 


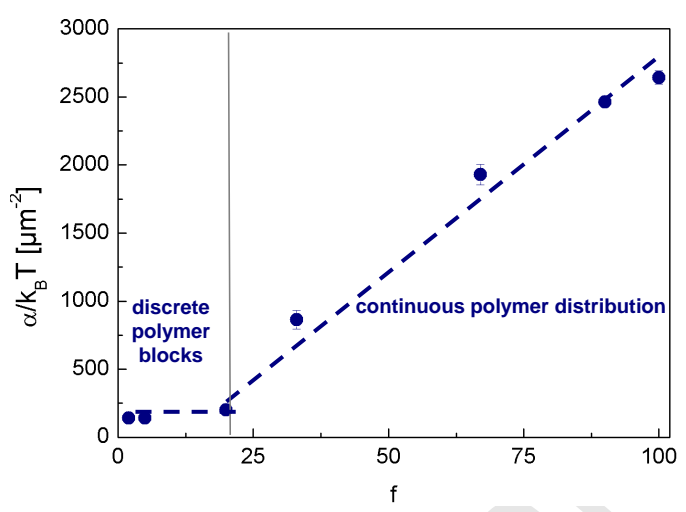

Figure 6: Coefficient $\alpha$, as shown in Eq 1 vs. PNIPAM surface fraction $f$.

a

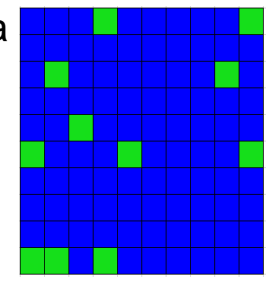

b

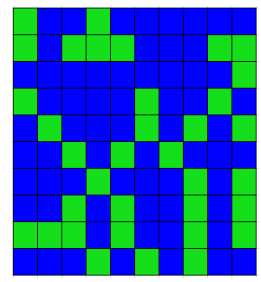

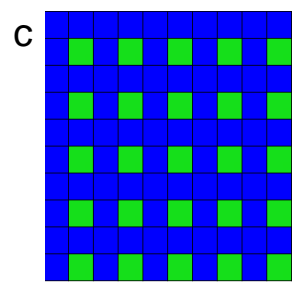

PLL-g-PEG
PLL-g-PNIPAM

Figure 7: Schematic views of possible polymer arrangements on bead surface. Each square represents a PLL-g-PEG (blue) or PLL-g-PNIPAM (green) block adsorbed on silica. a. Random configuration at $f=10 \%$ showing isolated PLL-g-PNIPAM blocks. b. Random configuration at $f=33 \%$ with continuous PLL-g-PNIPAM zones. c. Configuration possible at $f=25 \%$ explaining the approximate threshold between the two regimes by percolation arguments. 